Irish Math. Soc. Bulletin

Number 70, Winter 2012, 39-39

ISSN 0791-5578

\title{
ON PROPERTIES OF THE MIRIMANOFF POLYNOMIALS
}

\author{
DANIEL LYNCH
}

This is an abstract of the $\mathrm{PhD}$ thesis On Properties of the Mirimanoff Polynomials written by D. Lynch under the supervision of Prof Rod Gow at the School of Mathematical Sciences, University College Dublin and submitted in June 2012.

Historically the Mirimanoff polynomials were first considered in connection with solving Fermat's Last Theorem [1], and recently have been generalised further [2]. We show a surprising connection between these polynomials and the established theory of Costas arrays. Over finite fields, we investigate a conjectured bound on the number of roots. This is proven for some special cases. We also consider the polynomials over the complex domain, where it is shown that for odd $n$ (a parameter of the polynomial), the roots all occur in three distinct regions of the complex plane. We then build on these results to show the irreducibility of a specific factor of the Mirimanoff polynomials over the integer polynomial ring. Irreducibility is proven for $n=2 p$ and $n=3 p$ where $p$ is a prime. In addition, partial results are given for $n=5 p$ and $n=7 p$.

\section{REFERENCES}

[1] A. Cauchy, J. Liouville: Rapport sur un mémoire de M. Lamé relatif au dernier théorème de Fermat. JC. R. Acad. Sci. (9) 359-363 Paris. 1839.

[2] C. Helou: Cauchy-Mirimanoff Polynomials. C. R. Math. Rep. Acad. Sci. (2) 51-57 Canada. 1997.

School of Mathematical Sciences, University College Dublin

E-mail address, D. Lynch: dannylynch.com@gmail.com

2010 Mathematics Subject Classification. 12D10, $12 \mathrm{E} 05$.

Key words and phrases. Mirimanoff, polynomials, irreducibility.

Received on 12-12-2012.

Support from IRCSET and the Claude Shannon Institute is gratefully acknowledged. 